Analysis of Deformation Twinning in Tantalum Single Crystals Under Shock Loading Conditions

J. N. Florando, N. R. Barton, B. El-Dasher, J. M. McNaney, M. Kumar

December 17, 2012

Journal of Applied Physics 
This document was prepared as an account of work sponsored by an agency of the United States government. Neither the United States government nor Lawrence Livermore National Security, LLC, nor any of their employees makes any warranty, expressed or implied, or assumes any legal liability or responsibility for the accuracy, completeness, or usefulness of any information, apparatus, product, or process disclosed, or represents that its use would not infringe privately owned rights. Reference herein to any specific commercial product, process, or service by trade name, trademark, manufacturer, or otherwise does not necessarily constitute or imply its endorsement, recommendation, or favoring by the United States government or Lawrence Livermore National Security, LLC. The views and opinions of authors expressed herein do not necessarily state or reflect those of the United States government or Lawrence Livermore National Security, LLC, and shall not be used for advertising or product endorsement purposes. 


\section{Analysis of Deformation Twinning in Tantalum Single Crystals under Shock Loading Conditions}

Jeffrey N. Florando ${ }^{1, a)}$, Nathan R. Barton ${ }^{1}$, Bassem El-Dasher ${ }^{1}$, James M. McNaney ${ }^{1}$, and Mukul Kumar $^{1}$

${ }^{1}$ Lawrence Livermore National Laboratory, 7000 East Ave., Livermore, CA 94550

The competition between dislocation slip and twinning in tantalum single crystals has been investigated utilizing a crystal level twinning model and the results from gas gun recovery experiments conducted at peak normal stresses of 25 and $55 \mathrm{GPa}$. The recovered samples were characterized using electron back scattered diffraction, and the observed twining fractions were compared with the model. The experimental results show very low twin fractions in all orientations at $25 \mathrm{GPa}$; and that among (100), (110), (111), and (123) crystals the (110) crystals had the largest amount of twinning at 55GPa. The analysis shows that the general trends observed in the experimental data can be reproduced by the model when an orientation dependent dislocation evolution is used. This analysis gives insight into the possible influence of the dislocation density and its evolution on the observed twinning behavior.

\section{Introduction}

There have been a number of studies examining deformation twinning, and the review article by Christian and Mahajan ${ }^{1}$ is an excellent introduction to the topic. Many factors contribute to a material's propensity to deform by twinning. Materials with tetragonal or orthorhombic symmetry are more likely to deform via twinning than cubic symmetry materials, due to

\footnotetext{
a) Electronic mail: florando1@|lnl.gov
} 
relatively few easily activated slip systems and geometric considerations. Twinning has also been generally observed in the regime of very low temperatures, where the dislocation mobility is low, or high deformation rates, as for example, shock loading, where the applied stress rate outpaces the plastic relaxation from dislocation nucleation and multiplication ${ }^{2,3}$. Tantalum is one of the materials with cubic crystal symmetry for which twinning has been observed experimentally, and studies have shown that both polycrystalline and single crystal Ta deformed at liquid $\mathrm{He}$ (4.2 Kelvin) temperatures twin under quasi-static loading conditions ${ }^{4-6}$. Under shock loading there have been a number of studies where material has been recovered for post shock characterization $^{7-11}$. The general experimental observation is that under shock loading, twinning in Ta single crystals begins between a normal compressive stress of 7 to $14 \mathrm{GPa}$, however studies on polycrystalline material have also shown that much lower shear stress magnitudes can drive twin formation in tantalum. ${ }^{10,12}$ For single crystals, while there is evidence for an orientation dependence of the twinning behavior ${ }^{6,9}$, the exact nature of the dependence is still not fully understood.

In addition to the experimental observations, there have also been a number of constitutive models ${ }^{7,13,14}$ which have been developed specifically to describe the twinning behavior in Ta. Most of these models are designed to describe averaged polycrystalline behavior and take into account larger scale effects, such as the dependence on the grain size of the material.

In an effort to enhance the basic understanding of the competition between dislocation slip and twinning, a single crystal study has been conducted to try and avoid the complexities associated with the heterogeneous response of polycrystals. In this work, a crystal level twinning model has been developed and incorporated into a hydrodynamics code, and the simulation results are compared to experiments in which Ta single crystals have been shock loaded, 
recovered, and characterized using Electron Back Scattered Diffraction (EBSD). The crystal level twinning model provides a tool for assessing the impacts of various aspects of material physics in capturing the experimental observations and the underlying interaction between slip and twinning in tantalum.

\section{Experimental description}

Tantalum single crystal samples where the (100), (110), (111), or (123) planes were aligned normal to the impact direction were tested in the 2-stage gas gun facility at Lawrence Livermore National Laboratory. The samples were impacted at $800 \mathrm{~m} / \mathrm{s}$ and $1580 \mathrm{~m} / \mathrm{s}$, which generated a peak normal stress of 25 and $55 \mathrm{GPa}$, respectively. Details of the experiment are presented elsewhere $^{15}$.

After the samples were tested, the recovered samples were cut in half with a diamond saw, and the middle surfaces were metallographically polished and examined using EBSD. EBSD orientation mapping was carried out in a Philips XL30S FEG-SEM operating at $20 \mathrm{keV}$. All scans were performed at a magnification of 1,000X with a raster step size between 170 and 200 $\mathrm{nm}$. The probe beam was $\approx 5 \mathrm{~nm}$ in diameter, and given the sample inclination the probe dimensions would have been roughly elliptical (5 $\mathrm{nm}$ x $15 \mathrm{~nm})$. For each sample, the EBSD scan

size was approximately $25,000 \mu \mathrm{m}^{2}$. Since the scans were done at the higher magnification in an attempt to capture potentially small twins, it was difficult to generate enough scans to have statistical confidence in the area fraction of twins below a few percent. Therefore, fractions below this threshold are listed as less than $3 \%$. 


\section{Twinning Model}

A crystal level twinning model has been developed in an effort to describe the orientation dependence seen in the experimental data. In this model, the dislocations are assumed to slip on the twelve $\{110\}<111>$ slip systems, and twinning takes place on the twelve $\{211\}<111\rangle$ systems. For dislocation motion, the thermally activated portion has kinetics similar to the form found in the paper by Kothari et al. ${ }^{16}$, and the drag regime is based on molecular dynamics and dislocation dynamics calculations presented elsewhere ${ }^{17}$. The shearing rate on a give slip system (whether in the parent grain or in a twin variant) can be decomposed into a thermally activated portion and a drag limited portion, with the combined shearing rate

$$
\dot{\gamma}^{\alpha}=\left(\frac{1}{\dot{\gamma}_{\mathrm{w}}^{\alpha}}+\frac{1}{\dot{\gamma}_{\mathrm{r}}^{\alpha}}\right)^{-1} \text {. }
$$

The thermal activation portion follows the form

$$
\dot{\gamma}_{\mathrm{w}}^{\alpha}=\boldsymbol{\chi}_{\mathrm{wo}} h\left(-\exp \left(-\frac{\Delta G^{\alpha}\left(\tau^{\alpha}\right)}{\kappa \theta}\right)-\exp \left(-\frac{\Delta G^{\alpha}\left(-\tau^{\alpha}\right)}{\kappa \theta}\right)\right)
$$

where $\theta$ is the temperature, $h$ is the normalized dislocation density, and $\tau^{\alpha}$ is the resolved shear stress. Dependence on the resolved shear stress is similar to the MTS model ${ }^{18}$ and is modeled using

$$
\Delta G^{\alpha}\left(\tau^{\alpha}\right)=g_{0} b^{3} \mu^{\alpha}\left[1-\left(\frac{\tau^{\alpha}-g^{\alpha}}{\tau_{\mathrm{p}}}\right)^{p}\right]^{q} \quad \text { for } \quad \tau^{\alpha} \leq g^{\alpha}+\tau_{\mathrm{p}}
$$

where

$$
g^{\alpha}=g_{\mathrm{o}}+s \sqrt{h} .
$$


This form assumes that the Peierls barrier, $\tau_{\mathrm{p}}$, is the dominant thermally activated obstacle to dislocation motion, and the hardening effects due to dislocations are largely athermal.

In the phonon drag regime, slip is described by

$$
\dot{\gamma}_{\mathrm{r}}^{\alpha}=\left\{\begin{array}{cc}
\boldsymbol{\psi}_{\mathrm{ro}} h\left(1-\exp \left(-\frac{\left(\tau^{\alpha}-g^{\alpha}\right)}{D_{\mathrm{r}}}\right)\right) & \tau^{\alpha}>g^{\alpha} \\
0 & \tau^{\alpha} \leq g^{\alpha}
\end{array},\right.
$$

where $D_{r}$ is the drag stress, and based on molecular dynamics results ${ }^{17}$, is taken to be independent of temperature in the range of interest.

The model tracks a distinct scalar dislocation density within the parent grain and each of the twin variants, with the interaction among the parent and twin variants following the formalism described in the paper by Barton et al. ${ }^{19}$. The relative dislocation density evolves in a KocksMecking form ${ }^{20}$ as

$$
\dot{h}=\left(k_{1} \sqrt{h}-k_{2} h\right) \dot{\gamma} \text { where } k_{2}=\boldsymbol{\zeta}_{20}\left(\frac{\dot{\gamma}_{\mathrm{ko}}}{\dot{\gamma}}\right)^{1 / n} .
$$

Under this evolution form, the term with $\mathrm{k}_{1}$ describes dislocation multiplication and the term with $\mathrm{k}_{2}$ describes strain rate dependent annihilation. For a given strain rate, the dislocation density saturates at $\left(\mathrm{k}_{1} / \mathrm{k}_{2}\right)^{2}$ and the parameters are calibrated to capture the results of multiscale simulations presented by Barton et al. ${ }^{21}$. The shear strain rate, $\dot{\gamma}$, used in equation 6 is the cumulative shear rate calculated from the shearing rates from all of the slip systems associated with the given constituent (parent or twin variant). This averaged shear rate is only used to calculate how the dislocation density evolves. Therefore, orientation dependent behavior can still be captured through the orientation dependence of the shearing rates. Taken together, equations 4 and 6 result in the dislocation hardening being governed by a single scalar dislocation density within each constituent. While in principle a more generalized form could be 
developed to include the contributions from the density on each slip system, including latent hardening effects developed in other models ${ }^{22}$, for the purposes of this study, the averaged density appears sufficient to capture the overall behavior of the material. Further details and references related to a model with similar slip kinetics and hardening behavior are provided in another publication $^{23}$.

The relative dislocation density is a unitless quantity

$$
h \equiv \frac{\rho^{\perp}}{\rho_{o}^{\perp}}
$$

with $\rho^{\perp}$ being the actual number density of dislocations and $\rho_{o}^{\perp}$ being a reference value. For this work, $\rho_{o}^{\perp}$ has a value of $1 \times 10^{8} / \mathrm{cm}^{2}$, which is a common density for annealed single crystals. The parameters for the equations that describe the dislocation motion are provided in Table I.

The twinning kinetics follows a power law form as

$$
\dot{m}_{(i)}=\left\{\begin{array}{cl}
\Phi^{\mathrm{o}}\left(-\frac{f_{(i)}-\xi_{\mathrm{t}}}{g_{(i)}}\right)^{1 / r} & f_{(i)}>\xi_{\mathrm{t}(i)} \\
0 & f_{(i)} \leq \xi_{\mathrm{t}(i)}
\end{array}\right.
$$

Where $\mathrm{f}_{(\mathrm{i})}$ is the driving force (calculated from a projection of the stress and derivatives of the interaction energy described below), $\xi_{\mathrm{t}}$ is the threshold below which twins do not form, and $\mathrm{g}_{(\mathrm{i})}$ is the resistance to twinning and is assumed to obey the relationship

$$
g_{(i)}=\xi_{\mathrm{o}}+\xi \sqrt{h_{\eta_{\mathrm{fi}}(i)}}
$$


The resistance to twinning is dependent on the dislocation density of the constituent being swept by the twin boundary, , and the coefficient $\xi$ controls the degree to which the dislocations serve as obstacles to twinning. Given that the twins do not revert in the results shown here, in equation 9 is always the relative dislocation density in the parent grain. Having the resistance term in the denominator of equation 8 , implies that the dislocations serve as thermally activated barriers to twinning. It should be noted that the twinning equations mainly represent the growth of the twins, and do not have features specific to nucleation kinetics.

The interaction energy among the parent and twin variants influences coexistence of mass fractions at a given material point and takes the form

$$
E^{\mathrm{r}}=C^{\mathrm{r}} \mathrm{b}\left(1-m_{\mathrm{p}}\right)^{2}+\frac{1}{2} \sum_{\zeta, v=1}^{n_{\mathrm{c}}} m_{\zeta} A_{\zeta v}^{\mathrm{r}} m_{v}
$$

where $m_{p}$ is the fraction of the parent grain, $n_{c}=13$ for one parent and 12 twin variants, and

$$
A_{\zeta v}^{\mathrm{r}}=\left[\begin{array}{cc}
0 & 0 \cdots 0 \\
0 & \\
\vdots & C^{\mathrm{r}_{\mathrm{a}}}\left(1-\left|\mathbf{n}_{\zeta} \cdot \mathbf{n}_{\nu}\right|\right) \\
0 &
\end{array}\right] .
$$

The first term in equation 10 , with coefficient $C^{\mathrm{rb}}$, penalizes the overall formation of twins and the second term, with coefficient $C^{r a}$, penalizes the coexistence of twins in accordance with the degree to which their habit planes intersect. As seen in equation 10, it becomes harder to twin as the twin fraction increases.

The parameters for the twinning portion of the model are shown in Table I. This overall model is distinct from many other models in that it tracks both twin fractions and the slip behavior in the twinned portion of the material. By tracking the slip and twinning, the 
relationship between the dislocation kinetics and its connection to twinning can be studied in more detail. In addition, the functional forms and parameters for key features of the dislocation mechanics are motivated by sub-scale simulation results that are well suited to high strain rate behavior $^{21}$.

\section{Results and Discussion}

The EBSD results for the 25 GPa shot show very little twinning in all orientations, while the 55 GPa shot shows extensive twinning, particularly for the (110) crystal, as seen in Figure 1. The twin fractions for each orientation, summarized in Table II, were determined by summing the number of pixels that were within 5 degrees of the twin misorientation (60 degrees), and dividing by the total number of pixels in the scan. The corresponding (100) pole figures at 25 GPa, Figure 2, show very few peaks, indicative of only a few twin systems operating. At 55 GPa, the pole figures show large amounts of streaking, a sign of extensive deformation and rotation, and many more peaks caused by the activity of more twin systems.

The (110) oriented crystal shows no twinning at $25 \mathrm{GPa}$, yet shows the most twinning, nearly $25 \%$, at $55 \mathrm{GPa}$, while the (001) shows very little twinning under both conditions. The (111) and (123) orientation both show little twinning at $25 \mathrm{GPa}$, and 5-6\% at $55 \mathrm{GPa}$. While in principle EBSD has submicron resolution, it is possible that there are twins that are smaller than the EBSD resolution limit. TEM results from a previous study ${ }^{15}$ for the (100) $55 \mathrm{GPa}$ sample show twins that are only tens of nm in width, and hundreds of nanometers in length. Due to the spacing of the twins observed, it is estimated that any missed twin fractions are on the order of a few percent of the total area. 
In an effort to understand the observed experimental trends, the crystal level twinning model described above has been incorporated into a 3-D hydrodynamics code, and simulations of the plate impact experiments have been conducted. Since the EBSD scans are taken from the middle of the sample, periodic lateral boundary conditions are used. A montonic- $\mathrm{Q}^{24}$ is used so that the viscosity of the material model is dominant over the artificial viscosity in the plastic portion of the wave structure. For the twinning portion of the model, many of the parameters are difficult to measure experimentally; therefore the parameters reported have large uncertainties associated with them. Given these unknowns, the main value of this study is to see where the model is most sensitive in reproducing the trends observed in the experiments.

Previous studies have shown that there is a competition between dislocation slip and twinning $^{2,3,25,26}$, and many models account for this with a critical stress, where twinning is initiated once the local stress reaches a critical value $e^{7,14}$. The main limitation with such a model is that there is an inherent assumption that changes in the dislocation density do not affect the driving force required for twinning. Studies on polycrystalline material, however, have shown that twinning can be suppressed with prestrain ${ }^{2,3}$, indicating a strong dependence on the initial dislocation density. Using the model described in the previous section, a parameter study was conducted to evaluate which parameters most strongly influence the amount of twinning produced. Specifically, the role of the initial dislocation density, as well as the evolution of the dislocation density, through the dislocation multiplication rate, was studied.

\section{A. Dislocation density}

As shown in Table III, as the initial dislocation density, $\left.h\right|_{t=0}$, is increased in the simulation of the $55 \mathrm{GPa}$ shot, the amount of twinning decreases, from nearly $30 \%$ to $1-6 \%$. This 
observation is consistent with other experimental data that suggests twinning can be completely suppressed by pre-staining at low strain rates from a few percent to tens of percent depending on the deformation conditions ${ }^{1-3,25,26}$. In Figure 3 a, the simulated wave profile of the 55 GPa shot, using the parameters in Table I, are shown for a material point taken at the middle of the simulated sample. It is seen that in the (100) orientation, the longitudinal wave increases monotonically then begins to level off before slightly increasing again after about $0.35 \mu$ s. Also plotted are the distinct contributions to the effective strain from twinning versus dislocation slip at the same material point. The second rise in the longitudinal wave corresponds to an increase in the strain accommodated by the dislocations, which is delayed in time, and is consistent with the time dependence of dislocation motion and dislocation multiplication. For the (110) orientiation, Figure $3 b$, the strain due to twinning is significantly lower than in the (100) case, and the model predicts that eventually (after $0.3 \mu \mathrm{s}$ ) the strain due to dislocation slip will be higher than for twinning. This orientation also shows a drop in the particle velocity, which results from the complex interplay among the kinetics. At early times, twinning is the dominant plastic deformation mechanism and can be characterized as a rapid onset that has a tendency to saturate once the deformation can be accommodated by dislocation slip. Under certain conditions, the kinetic interactions are such that the wave can separate to have two distinct rises, with material plastically relaxing in between. Since the particle velocity is governed by the derivative of the normal stress with respect to volume, a drop in particle velocity represents a relaxation in the deviatoric stress, which can launch rarefaction waves that partially unload the shock wave. The pullback feature in the particle velocity can be viewed as a superposition of these effects, and has been reported in other studies on shocked $\mathrm{Ta}^{27}$. 
Regarding the dislocation evolution, the assumption is that the dislocation density is increasing due to multiplication from existing sources, as opposed to the homogenous nucleation of dislocations. Utilizing molecular dynamics (MD) simulations, Rudd et al. showed that in Ta, homogenous nucleation is expected to occur at normal stresses above $62 \mathrm{GPa}$ under laser driven conditions, which is a higher normal stress and strain rate than observed in these experiments ${ }^{28}$.

When another simulation is performed with the initial dislocation density increased by a factor of 100, which would correspond to a heavily deformed initial condition of the sample, the results for both orientations, Figures $3 \mathrm{c}$ and $3 \mathrm{~d}$, show a sharp increase in the particle velocity in the first rise, before leveling off with no additional rises. In this case, the simulation shows that the strain is being mainly accommodated by dislocation slip, since the dislocation density is sufficiently high. In examining equations 2 and 4, the strain rates due to slip are a strong function of the dislocation density, therefore, these results are consistent with the theory that if there are enough dislocations to accommodate the strain rate seen, and thereby prevent overshoot in the deviatoric stress magnitude, then twinning will be suppressed.

\section{B. Dislocation multiplication rate}

The dislocation multiplication rate can also have a large effect on the amount of twinning predicted. For these simulations the initial dislocation density is fixed at $\mathrm{h}=1$, which is consistent with TEM observations of the initial dislocation density of annealed bcc single crystals. ${ }^{29}$ The simulation results, Tables IV and $\mathrm{V}$, show that for a given orientation, as the multiplication rate increases, the corresponding twin fraction decreases. The effect is similar to the increase in the initial dislocation density, where the multiplication rate is sufficient to create enough dislocations to accommodate the straining at the relevant rates. In these results however, for a given 
multiplication rate, the orientation dependence appears to be inconsistent with the trends observed experimentally. Mainly, the (100) orientation appears to have the largest twin fraction. Unlike the initial dislocation density however, which is basically the same for all of the orientations, the multiplication rate can depend on the orientation. Single crystal experiments on (100), (110) and (111) single crystals show that the strain hardening rate is markedly different for each orientation, ${ }^{30,31}$ where the (100) orientation shows the higher strain hardening rate, (110) has the lowest rate, and the (111) orientation is somewhere between the two. Given that strain hardening can be attributed to the interaction of dislocations, there is generally a direct correlation between the strain hardening rate and the dislocation multiplication rate. Using a dislocation dynamics code, Bulatov et al., found that the multiplication rate from the (100) orientation is an order of magnitude higher than for the (110) orientation ${ }^{32}$. They attribute the difference to special dislocation junctions that form in the (100) case that act as sources which increase the multiplication rate, and also show an increase in the hardening.

Based on these cases the multiplication rate, $\mathrm{k}_{1}$, was varied based on the orientation, where the (110) orientation had the lowest rate, approximately 150 , the (100) orientation had the highest rate about 8000 , and the (111) and (123) orientations were set at approximately 800 . It should be noted that the annihilation rate $\mathrm{k}_{2}$, is also changed such that the ratio of $\mathrm{k}_{1}$ to $\mathrm{k}_{2}$ squared is consistent with the saturation density from the LLNL Ta multiscale model ${ }^{21}$. Using these parameters for $\mathrm{k}_{1}$ and $\mathrm{k}_{2}$, the experimental trends are reproduced, shown as the highlighted values in Tables IV and V, where the (110) orientation shows a considerably larger amount of twinning than the other orientations under a $55 \mathrm{GPa}$ normal stress loading. While the amount of twinning in the (100) orientation is higher than experimentally observed, it is possible that there are fine scale twins that are missed in the ESBD analysis. The implication from these results is 
that the multiplication rate plays a prominent role in determining whether a given orientation, or grain, will twin.

While similar trends appear in the experimental characterization and the model results, there are still some discrepancies that imply that the models are missing relevant aspects of the physics. One source of improvement could include results from MD simulations to guide the

development of twinning kinetic equations that incorporate both nucleation and growth ${ }^{33}$. Since it appears that the dislocation evolution is important to twinning, it would be expected that the results would be sensitive to the nucleation and growth kinetics of the twining as well. Another source of improvement could be to develop a model to resolve the individual twin domain formation instead of relying on the interaction energy model and homogenization over twin fractions. It should also be noted that the interactions between the twinning and dislocation evolution is expected to be strain rate dependent, and that for lower strain rates different aspects of the material response may be dominant, although the interaction between slip and twinning is likely to still be complex.

\section{Conclusions}

A crystal level model has been created which tracks the deformation contribution from the individual dislocation and twinning systems. This model has been incorporated into a hydrodynamic code that is used to simulate gas gun experiments on differently oriented single crystals. The experimental trends in the orientation dependence of the twinning fraction are captured with the model when an orientation dependence on the dislocation multiplication rate is invoked. The (110) oriented sample was found to have a substantially larger fraction of twins at $55 \mathrm{GPa}$, in comparison to the other orientations, and the model captured this trend when the 
multiplication rate of the (110) crystal is an order of magnitude less than the (100) orientation.

This dependence is consistent with other studies on the strain hardening and multiplication rates in Ta single crystals.

\section{Acknowledgements}

The authors would like to thank Dr. Ricky Chau for the gas gun recovery experiments, Dr.

Bryan Reed for discussions of the interpretation of the particle velocity simulation results,

Jackson Go for sample preparation, and Edwin Sedillo for operating the SEM. Finding from the National Nuclear Security Administration (NNSA) “Dynamic Materials Properties” science campaign is gratefully acknowledge. This work was performed under the auspices of the U.S. Department of Energy by Lawrence Livermore National Laboratory under Contract DE-AC5207NA27344.

\section{References}

${ }^{1}$ J. W. Christian, and S. Mahajan, Prog. Mater. Sci. 39(1-2), 1, (1995).

${ }^{2}$ S. Mahajan, Phys. Status Solidi (b) 33(1), 291, (1969).

${ }^{3}$ S. Mahajan, and A. Bartlett, Acta Metall. 19(10), 1111, (1971).

${ }^{4}$ T. Mitchell, and W. Spitzig, Acta Metall. 13(11), 1169, (1965).

${ }^{5}$ J. Shields, S. Goods, R. Gibala, and T. Mitchell, Mater. Sci. and Eng. 20,71, (1975).

${ }^{6}$ P. Sherwood, F. Guiu, H. Kim, and P. Pratt, Can.. Journ. of Phys. 45(2), 1075, (1967).

${ }^{7}$ L. Murr, M. Meyers, C. S. Niou, Y. Chen, S. Pappu, and C. Kennedy, Acta Mater. 45(1), 157, (1997).

${ }^{8}$ D. Lassila, and G. GRAY III, Le Journ. de Phys. IV 1(C3), 3-8, (1991).

${ }^{9}$ L. Hsiung, and D. Lassila, Acta Mater. 48(20), 4851, (2000).

${ }^{10}$ G. T. R. Gray III, V. Livescu, E. Cerreta, T. Mason, P. Maudlin, and J. Bingert, in DYMAT 2009:

Proccedings of the 9th International Conference on the Mechanical and Physical Behaviour of Materials under Dynamic Loading, Brussels, Belgium, September 7-11 2009,(EDP Sciences), 2,pp. 963-969.

${ }^{11}$ G. Gray, N. Bourne, and J. Millett, J. Appl. Phys. 94(10), 6430, (2003).

${ }^{12}$ B. W. Reed, J. Reed Patterson, D. C. Swift, J. S. Stolken, R. W. Minich, and M. Kumar, J. Appl. Phys. 110(11), 113506, (2011).

${ }^{13}$ F. Zerilli, and R. Armstrong, Le Journ. de Phys. IV 7(C3), 3-3, (1997).

${ }^{14}$ M. Meyers, O. Vohringer, and V. Lubarda, Acta Mater. 49(19), 4025, (2001).

${ }^{15}$ J. McNaney, L. Hsuing, N. Barton, and M. Kumar, AIP Conf. Proc. 1195,1127, (2009). 
${ }^{16}$ M. Kothari, and L. Anand, J. Mech. and Phys. of Solids 46(1), 51, (1998).

${ }^{17}$ N. Barton, A. Arsenlis, M. Rhee, J. Marian, J. V. Bernier, M. Tang, and L. Yang, AIP Conf. Proc. 1426(1), 1513, (2012).

${ }^{18}$ P. Follansbee, and U. Kocks, Acta Metallurgica 36(1), (1988).

${ }^{19}$ N. Barton, D. Benson, and R. Becker, Mod. Sim. in Mat. Sci. and Eng. 13(5), (2005).

${ }^{20}$ H. Mecking, and U. Kocks, Acta Metall. 29(11), 1865, (1981).

${ }^{21}$ N. Barton, J. Bernier, R. Becker, A. Arsenlis, R. Cavallo, J. Marian, M. Rhee, H. S. Park, B. Remington, and R. Olson, J. Appl. Phys. 109,073501, (2011).

${ }^{22}$ R. J. Asaro, and A. Needleman, Acta Met. 33(6), 923, (1985).

${ }^{23}$ N. R. Barton, N. W. Winter, and J. E. Reaugh, Mod. Sim. in Mat. Sci. and Eng. 17(3), (2009).

${ }^{24}$ M. L. Wilkins, J. Comp. Phys. 36(3), 281, (1980).

${ }^{25}$ A. Churchman, and A. Cottrell, Nature 167,943, (1951).

${ }^{26}$ N. Boucher, and J. Christian, Acta Metall. 20(4), 581, (1972).

${ }^{27}$ J. Asay, T. Ao, T. Vogler, J. P. Davis, and G. Gray, J. Appl. Phys. 106(7), 073515, (2009).

${ }^{28}$ R. E. Rudd, A. Comley, J. Hawreliak, B. Maddox, H. S. Park, and B. A. Remington, AIP Conf. Proc. 1426,1379, (2012).

${ }^{29}$ M. Rhee, D. H. Lassila, V. V. Bulatov, L. Hsiung, and T. D. de la Rubia, Phil. Mag. Lett. 81(9), 595, (2001).

${ }^{30}$ J. Byron, J. Less Comm. Metals 14(2), 201, (1968).

${ }^{31}$ D. Rittel, M. Silva, B. Poon, and G. Ravichandran, Mech. Mater. 41(12), 1323, (2009).

${ }^{32}$ V. V. Bulatov, L. L. Hsiung, M. Tang, A. Arsenlis, M. C. Bartelt, W. Cai, J. N. Florando, M. Hiratani, M. Rhee, and G. Hommes, Nature 440(7088), 1174, (2006).

${ }^{33}$ L. A. Sandoval, M. P. Surh, A. A. Chernov, and D. F. Richards, submitted to Acta Mat. (2012).

${ }^{34}$ D. L. Preston, D. L. Tonks, and D. C. Wallace, J. Appl. Phys. 93,211, (2003).

${ }^{35}$ W. F. Hosford, Oxford University Press(USA), 1993, 248, (1993).

${ }^{36}$ S. R. Chen, and G. T. Gray, Metall. and Mater. Trans. A 27(10), 2994, (1996).

${ }^{37}$ D. Steinberg, Technical Report UCRL-MA-106439, Lawrence Livermore National Laboratory, (1996). 
Tables and Figures

Table I. Model parameters

\begin{tabular}{|c|c|c|c|c|}
\hline Parameter & Value & Name & Equation & Reference(s) \\
\hline$g_{\circ} b^{3} / \kappa$ & $23034.4 \frac{\mathrm{K}}{\mathrm{M} b a r}$ & activation energy parameter & 3 & 16 \\
\hline$p$ & 0.28 & MTS curve shape parameter & 3 & 16 \\
\hline$q$ & 1.34 & MTS curve shape parameter & 3 & 16 \\
\hline$\tau_{\mathrm{p}}$ & $400 \mathrm{MPa}$ & Peierls stress & 3 & 16 \\
\hline$\dot{\gamma}_{\text {wo }}$ & $2 \cdot 10^{7} \mathrm{~s}^{-1}$ & ref. thermally activated slip rate & 2 & 16 \\
\hline$\dot{\gamma}_{\mathrm{r} 0}$ & $1 \cdot 10^{3} \mu \mathrm{s}^{-1}$ & ref. drag limited slip rate & 5 & 34 \\
\hline$D_{\mathrm{r}}$ & $0.02 \mathrm{M} b a r$ & reference drag stress & 5 & \\
\hline$\theta_{\mathrm{o}}$ & $300 \mathrm{~K}$ & reference temperature & 5 & \\
\hline$k_{1}$ & 160 & D.D. production constant & 6 & \\
\hline$k_{20}$ & 10 & D.D. production constant & 6 & \\
\hline$g_{\text {o }}$ & $\mathrm{OMPa}$ & slip resistance constant & 4 & \\
\hline$s$ & $49 \mathrm{MPa}$ & slip resistance constant & 4 & \\
\hline$c_{11}$ & $260.2 \mathrm{GPa}$ & elastic moduli & & 16,35 \\
\hline$c_{12}$ & 154.5GPa & elastic moduli & & 16,35 \\
\hline$c_{44}$ & $82.6 \mathrm{GPa}$ & elastic moduli & & 16,35 \\
\hline$c_{\mathrm{v}}$ & $0.1455 \frac{\mathrm{kJ}}{\mathrm{kg} \cdot \mathrm{K}}$ & heat capacity & & 36 \\
\hline$\rho_{\mathrm{o}}$ & $16.6 \frac{\mathrm{g}}{\mathrm{cm}^{3}}$ & reference density & & 36 \\
\hline$S$ & 1.2 & EOS & & 37 \\
\hline$\Gamma_{0}$ & 1.67 & EOS & & 37 \\
\hline$a$ & 0.42 & EOS & & 37 \\
\hline$\xi_{\mathrm{t}}$ & $300 \mathrm{MPa}$ & twin threshold & 8 & 7 \\
\hline$\xi_{0}$ & $400 \mathrm{MPa}$ & twin resistance constant & 8,9 & \\
\hline$\xi$ & $80 \mathrm{MPa}$ & twin resistance constant & 8,9 & \\
\hline$r$ & 0.1 & twin rate sensitivity & 8 & \\
\hline$\dot{\Phi}^{\circ}$ & $1.25 / \mathrm{s}$ & reference twinning rate & 8 & \\
\hline $\mathrm{C}^{\mathrm{ra}}$ & $300 \mathrm{MPa}$ & twin penalty constant & 10,11 & \\
\hline $\mathrm{C}^{\mathrm{rb}}$ & $700 \mathrm{MPa}$ & twin penalty constant & 10 & \\
\hline
\end{tabular}


Table II. Twin fractions measured from EBSD scans.

\begin{tabular}{|c|c|c|c|c|}
\hline Normal Stress & $(100)$ & $(110)$ & $(111)$ & $(123)$ \\
\hline $25 \mathrm{GPa}$ & $<3 \%$ & $<3 \%$ & $<3 \%$ & $<3 \%$ \\
\hline $55 \mathrm{GPa}$ & $<3 \%$ & $25 \%$ & $6 \%$ & $5 \%$ \\
\hline
\end{tabular}

Table III. Effect of initial dislocation density on the twin fraction for the $55 \mathrm{GPa}$ simulation

\begin{tabular}{|c|c|c|}
\hline $\begin{array}{c}\text { Initial relative dislocation } \\
\text { density (h) }\end{array}$ & $(100)$ & $(110)$ \\
\hline 1 & $29 \%$ & $29 \%$ \\
\hline 100 & $7 \%$ & $1 \%$ \\
\hline
\end{tabular}

Table IV. Simulation results of the twin fraction for the $25 \mathrm{GPa}$ simulation

\begin{tabular}{|c|c|c|c|c|}
\hline Multiplication rate, $\mathrm{k} 1$ & $(100)$ & $(110)$ & $(111)$ & $(123)$ \\
\hline 150 & $6.6 \%$ & $2.1 \%$ & $1 \%$ & $2.9 \%$ \\
\hline 800 & $1.9 \%$ & $0.1 \%$ & $0.1 \%$ & $0.5 \%$ \\
\hline 8000 & $0 \%$ & $0 \%$ & $0 \%$ & $0 \%$ \\
\hline
\end{tabular}

Table V. Simulation results of the twin fraction for the 55GPa simulation

\begin{tabular}{|c|c|c|c|c|}
\hline Multiplication rate, k1 & $(100)$ & $(110)$ & $(111)$ & $(123)$ \\
\hline 150 & $24.7 \%$ & $23.4 \%$ & $24.3 \%$ & $24.5 \%$ \\
\hline 800 & $15.2 \%$ & $1.1 \%$ & $0.8 \%$ & $1.9 \%$ \\
\hline 8000 & $7.1 \%$ & $0.7 \%$ & $0.2 \%$ & $1.4 \%$ \\
\hline
\end{tabular}



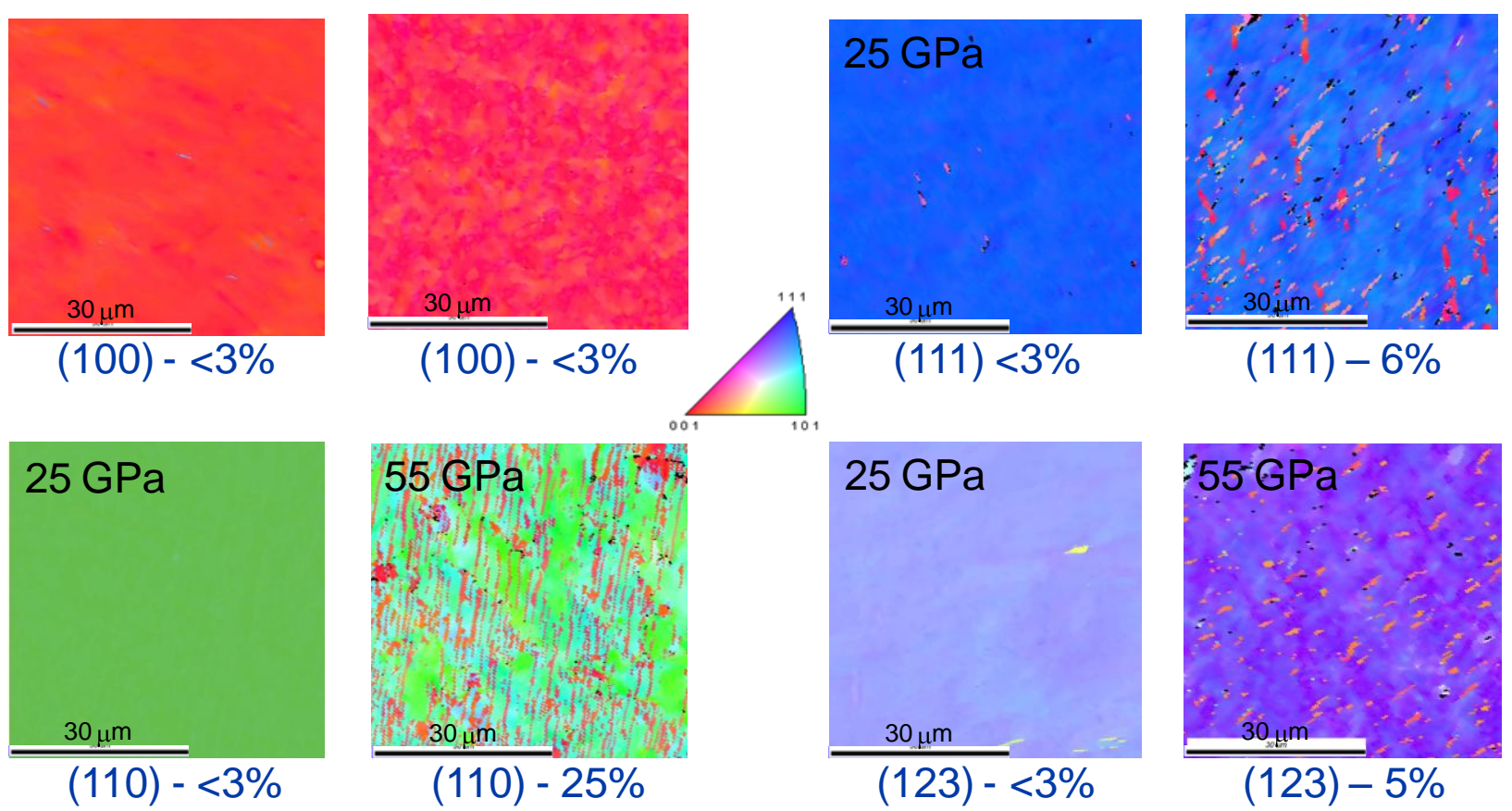

Figure 1- EBSD orientation map results for the 25 and 55 GPa case for each orientation ${ }^{15}$. The (110) orientation shows extensive twinning at $55 \mathrm{GPa}$.
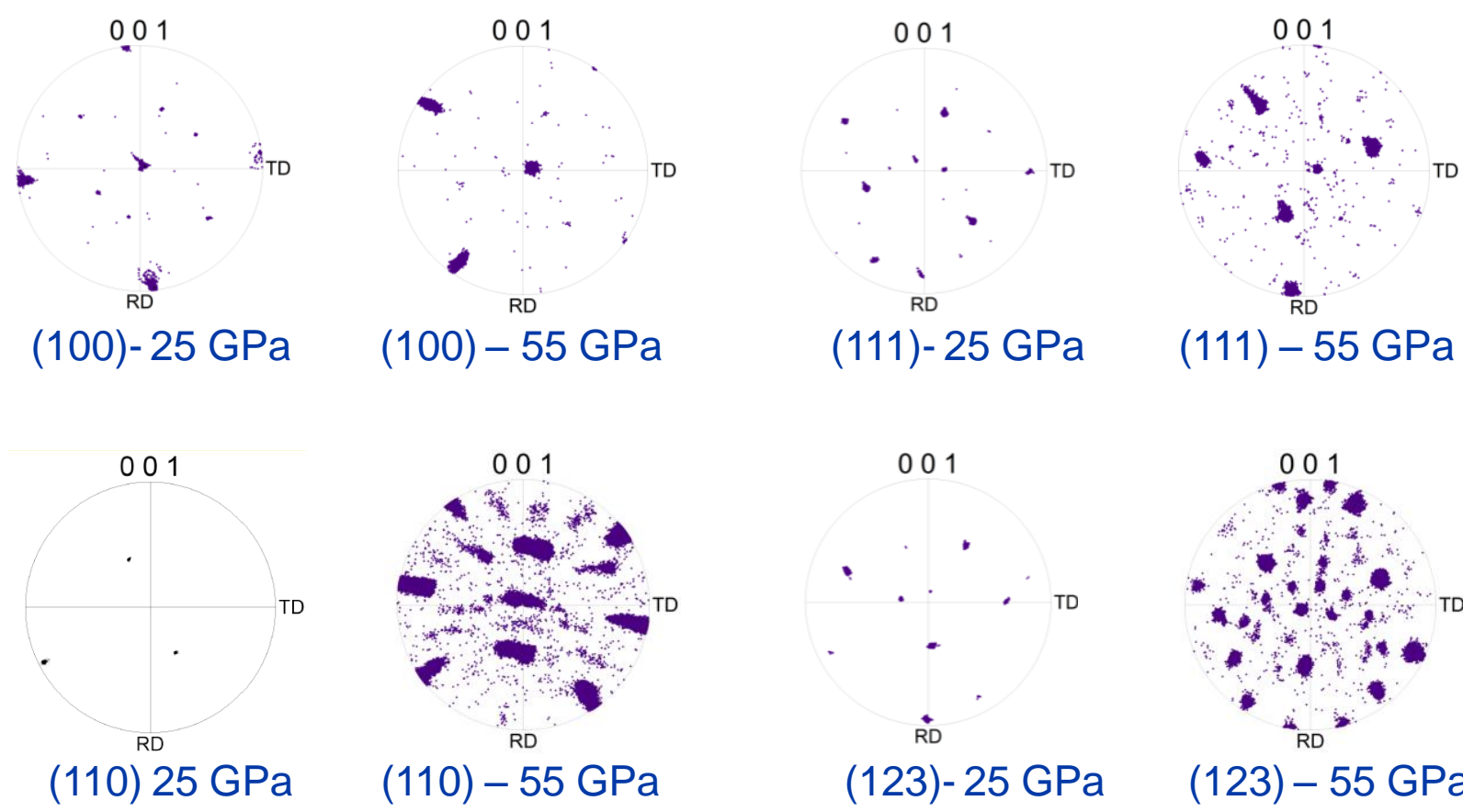

(111)- $25 \mathrm{GPa}$

(111) $-55 \mathrm{GPa}$

(110) $25 \mathrm{GPa}$
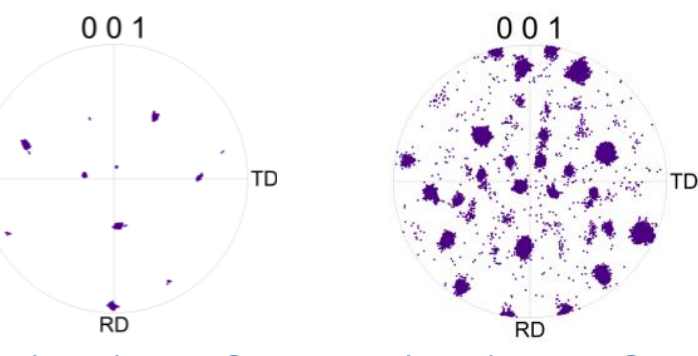

(123)- $25 \mathrm{GPa}$

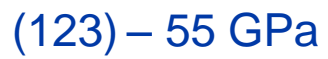

Figure 2- (100) pole figures for $25 \mathrm{GPa}$ and $55 \mathrm{GPa}$ cases $^{15}$. The additional spots indicate activity on twin systems. 

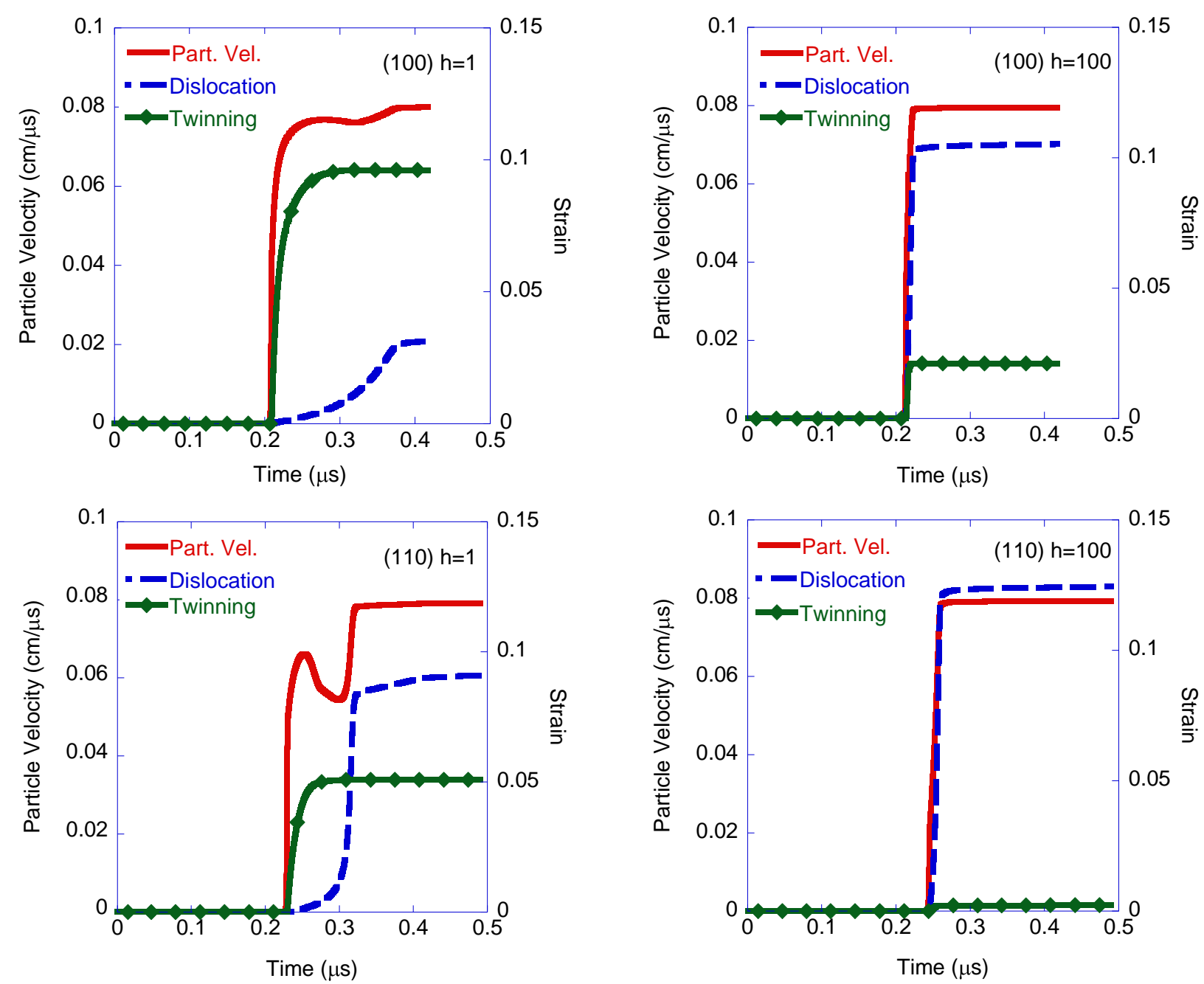

Figure 3 - Simulated longitudinal particle velocity and fractional strain plots for the (100) and (110) orientations using different initial dislocations densities, $h$, a) (100) orientation for $\mathrm{h}=1, \mathrm{~b})(110)$ orientation for $h=1, c)(100)$ orientation for $h=100, d)$ (110) orientation for $h=100$. 